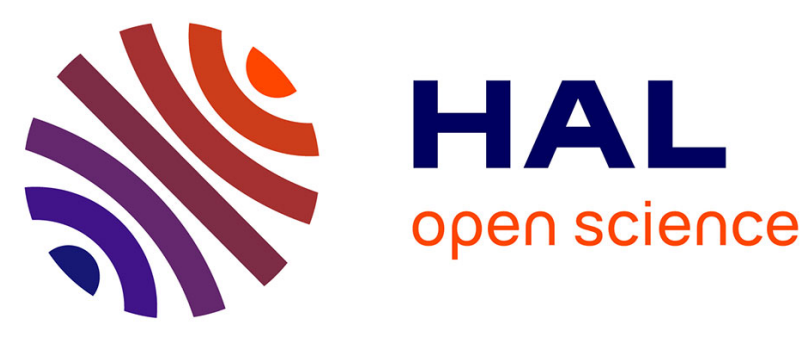

\title{
Analysis and performance evaluation of the next generation wireless networks
}

\author{
Arash Maskooki, Gabriele Sabatino, Nathalie Mitton
}

\section{To cite this version:}

Arash Maskooki, Gabriele Sabatino, Nathalie Mitton. Analysis and performance evaluation of the next generation wireless networks. Modeling and Simulation of Computer Networks and Systems: Methodologies and Applications, 2015, 2015, 10.1016/B978-0-12-800887-4.00021-3 . hal-01149044

\section{HAL Id: hal-01149044 \\ https://hal.inria.fr/hal-01149044}

Submitted on 13 Nov 2015

HAL is a multi-disciplinary open access archive for the deposit and dissemination of scientific research documents, whether they are published or not. The documents may come from teaching and research institutions in France or abroad, or from public or private research centers.
L'archive ouverte pluridisciplinaire HAL, est destinée au dépôt et à la diffusion de documents scientifiques de niveau recherche, publiés ou non, émanant des établissements d'enseignement et de recherche français ou étrangers, des laboratoires publics ou privés. 


\title{
Analysis and performance evaluation of the next generation wireless networks
}

Arash Maskooki, Gabriele Sabatino, Nathalie Mitton

Inria Lille-Nord Europe, Villeneuve d'Ascq, France. Email: firstname.lastname@inria.fr

\begin{abstract}
Exponential growth in demand for high bandwidth applications such as multimedia in cellular networks has triggered the need for development of new technologies capable of providing the required high bandwidth, reliable links in wireless environments. However, the available spectrum is a limited natural resource and the current technologies have been shown to have low spectrumefficiency. To improve the spectrum efficiency, heterogeneous networks are considered as a viable solution. They are composed of a mix of macro, pico, femto and relay base-stations that improve the spectrum efficiency and throughput performance of the system. The aim of this chapter is to provide an overview of these emerging technologies. This chapter describes the main characteristics and performance requirements that the next generation networks must fulfill. Particularly, the focus is on Long Term Evolution (LTE)/LTE-Advanced technologies where some possible improvements and challenges are explained. Subsequently, the analytical methods and simulations techniques to evaluate the performance of the next generation heterogeneous networks are discussed. Finally, the simulation results for some example scenarios are provided and discussed.
\end{abstract}

\section{Introduction}

The data traffic consumption is exponentially growing in wireless networks; this is due to the new applications demanding high-quality of services, high data rate and new advanced user terminals which are evolving rapidly over the past decade.

Mobile data traffic is forecast to grow by more than 24 times between 2010 and 2015, and 500 to 1000 times between 2010 and 2020 [1]. The forecasts estimate that about 50 billion devices will be connected by 2020 .

Long-Term Evolution/Long-Term Evolution-Advanced (LTE/LTE-A) wireless systems as well asIEEE802.11 family of technology are expected to dominate the wireless-communication arena for the next decade.Particularly,LTE/LTE-Aare more and more emerging as the global choice which all mobile-broadband operators are expected to migrate [2-3].

Other radio access technologies are available or under development such as Wireless Personal Area Network (WPAN includingBluetooth and ZigBee) and IEEE802.11p, for very-short-range communication between devices and for communication between vehicles.

The vision of new generation mobile systems is towards unification of various mobile and wireless networks which can be qualified as heterogeneous network (HetNet). HetNets consist of different access networks and provide a wide range of services including enhanced and extended mobility and accessibility. 
These networks will not only help improving existing services [4-5] but integrate intelligent algorithms for mobility management, resource management, access control, routing, etc. This chapter will discuss modeling and analysis of the performance of the next generation wireless networks. This section provides an introduction to this chapter. The following section will discuss the characteristics and development of different generations of cellular wireless network.Section 3 will present the analytical methods to evaluate the performance of the next generation HetNets. Subsequently, Section 4 will discuss the simulation techniques and analysis for HetNets. Finally, Section 5 will conclude this chapter.

\section{The evolution of cellular wireless systems}

The First Generation (1G) mobile cellular systems were deployed in the 1980s. The first commercial cellular network was the Nordic Mobile Telephone (NMT) deployed in the Scandinavian countries. Another system developed was the advanced mobile phone service (AMPS) cellular system in the United States and the most popular Total Access Communication Systems (TACS). These technologies were based on analog systems, circuit switching and used Frequency Division Multiple Access (FDMA) radio system.

Second generation (2G) mobile systems were introduced at the beginning of the 1990s in Europe with the Global System for Mobile communication (GSM) systems. The GSM system, developed by the European Telecommunications Standard Institute (ETSI), is a Time Division Multiple Access (TDMA) radio system with data transmission rate up to $9.6 \mathrm{kbps}$ and is the pioneer of the packet switching system.

In United States there were other lines of development such as IS-54 (North America TDMA Digital Cellular), IS-136 also known as D-AMPS and IS-95 based on Code Division Multiple Access (CDMA) known as CDMA-One.

To provide better support for data services, ETSI developed the General Packet Radio Service (GPRS), a packet transmission system that overlays GSM and inter-works with external packet data networks such as the Internet. GPRS is a 2.5 generation (2.5G) wireless communication system. The main feature is that each mobile terminal is assigned an IP address which enables the devices in such networks to integrate with Internet easily.

The evolution towards third generation cellular systems (3G) was driven by the International Telecommunications Union (ITU) and referred to as International Mobile Telecommunications 2000 (IMT-2000).

The Third Generation Partnership Project (3GPP), organization established in 1998, defined a mobile system called universal mobile telecommunications systems (UMTS).Its mainfeature was to evolve GSM core networks and the Radio Access Network (RAN) technologies. 3GPP 
developed a system that enhanced data rates for GSM evolution (EDGE) standards. The $3 G$ systems can deliver bit rates up to $2 \mathrm{Mbps}$ and support of quality-of-service (QoS). The approach adopted to evolve the core network is called High Speed Packet Access (HSPA) and is widely considered as $3.5 \mathrm{G}$. The HSDPA offered peak rate of $14.4 \mathrm{Mbps}$ in $5 \mathrm{MHz}$ channel at the downlink.

The 3GPP started to work on the Fourth Generation (4G) cellular systems and standardized the Long-Term Evolution (LTE)[6]. LTE in effect was labeled 3.9G and the motivation behind the design of this network was to provide low latency and very high-data rates starting from at 100 Mbps and reaching more than $1 \mathrm{Gbps}$ at the downlink.

LTEis expected to substantially improve the end-user throughputs, sector capacity and reduce user plane latency, bringing significantly improved user experience with full mobility. LTE is scheduled to provide support for IP-based traffic with end-to-end QoS and in particular, also LTE supports a flexible bandwidth deployment and, thanks to Orthogonal Frequency Division Multiplexing (OFDM) and Multiple Input Multiple-Output (MIMO) systems, allows high data rates.

Enhancements introduced in LTE are [6]:

- Downlink peak data rate up to $100 \mathrm{Mbps}$;

- Uplink peak data rate up to $50 \mathrm{Mbps}$;

- Increased data rate for cell-edge users with respect to HSPA;

- High spectral efficiency (bit/s/Hz), increased by a factor 3-4 with respect to HSPA;

- Round Trip Time (RTT) equals to 10 ms (it was 70 ms in HSPA and 200 ms in UMTS);

- Low latency, lower than 100 ms for idle-to-active mode switch;

- Mobility support for very high mobility speed (up to $350 \mathrm{~km} / \mathrm{s}$ );

- Backward compatibility (GSM/GPRS and UMTS/HSPA);

- Voice and real time services offered over IP network.

These enhancements are supported by several features [6]:

- OFDM/OFDMA downlink modulation;

- SC-FDMA uplink modulation;

- High channel bandwidth flexibility $(1.4,3,5,10,15,20 \mathrm{MHz})$ both in downlink and uplink direction; 
- Very large section of available spectrum can be assigned to a LTE base station (including GSM or UMTS frequencies or band on $2.6 \mathrm{GHz}$ );

- Transmitting and receiving diversity, spatial multiplexing and diversity with Multiple Input Multiple Output (MIMO) techniques;

- QPSK, 16-QAM and 64-QAM modulation schemes.

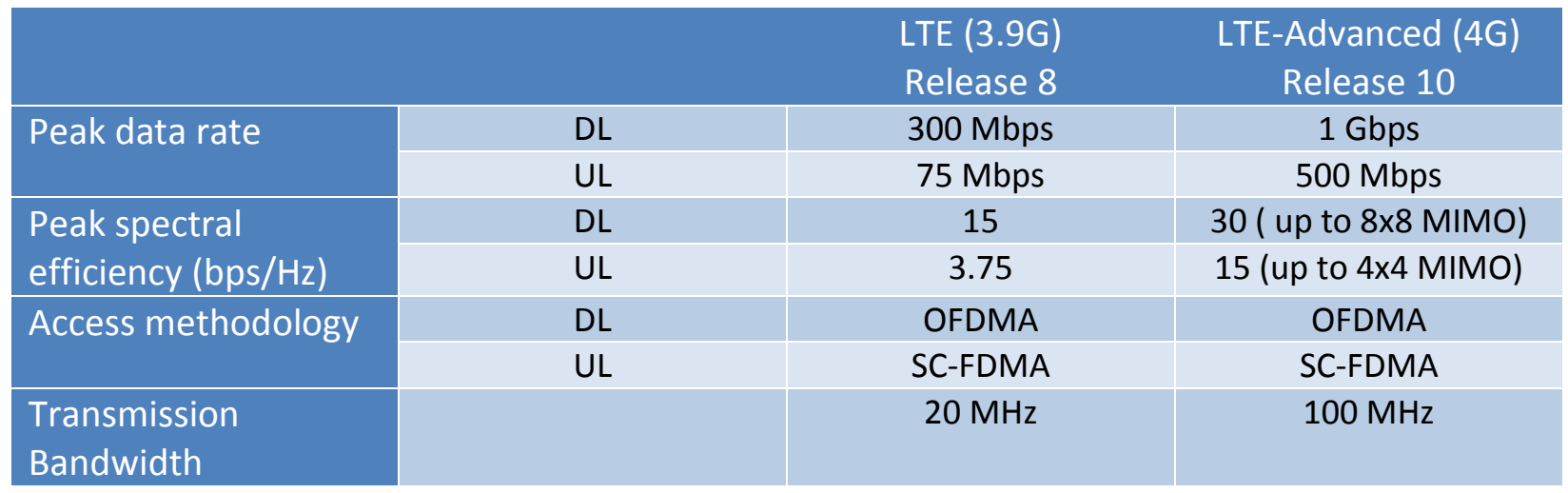

Table 1: Performance LTE and LTE-Advanced.

The main goals of LTE are focused on minimizing system and User Equipment (UE) complexities, allowing flexible spectrum deployment in existing or new frequencyspectrum management and enabling co-existence with other 3GPP Radio Access Technologies (RATs).

The definition of $4 \mathrm{G}$ wireless, known as the International Mobile Telecommunications Advanced (IMT-Advanced) project, was finally published by the International Telecommunications Union Radio communication Sector (ITU-R) in March 2008. In 2009 LTEAdvanced (LTE-A) (in Release 10), the 4 G mobile evolution system, was defined.

LTE-A research is mainly focused on user Signal to Interference Noise Ratio (SINR) improvement and spectrum flexibility. The LTE-A proposes a distributed network architecture, heterogeneous networkwhich consist of a mix of macro-, pico-, femto-cells (HeNBs - Home eNodeB - also called femto-cells, low cost eNodeBs for indoor coverage improvement), and relay base-stations. They can be directly connected to the Evolved Packet Core (EPC) or via a gateway (HeNB-GW - Home eNodeB Gateway), providing additional support for a large number of HeNBs. Self-Organizing Network (SON) concepts have been introduced in LTE standardization in order to increase the network performance and reduce the operational expenditure for operators. The smaller transmission powers from small-cells and the relative proximity of users to a small-cell, more users can now be covered within the same area in a HetNet; consequently, HetNets provide "cell-splitting" gains relative to macro-only networks.

The new technology targets include,better coverage, higher data rates, better QoS performance and fairness among users. 3GPP has been working on various aspects to improve LTE 
performance in the framework of LTE-Advanced, which includes higher order MIMO, Carrier Aggregation and Cooperative Multipoint Transmission (CoMP). Carrier Aggregation consists of grouping several LTE Component Carriers (CC), so that devices are able to use bandwidth up to 100 Mhz. Carrier Aggregation can be implemented by different approaches. The first one consists of contiguous bandwidth, where five contiguous $20 \mathrm{MHz}$ channels are aggregated to obtain the required bandwidth. The other approachis non-contiguous carrier aggregation. In this case, CC can be non-contiguous on the same spectrum band or non-contiguous on different spectrum band. In LTE-Advanced design, support of multiple antenna system is necessary to achieve data rates of $1 \mathrm{Gbps}$ in downlink and $500 \mathrm{Mbps}$ in uplink within a bandwidth of $20 \mathrm{MHz}$. The key requirement for an LTE mobile station is to use 2 antennas for uplink and 2 antennas for downlink. The concept of multiple antennas became popular to increase throughputs along with adaptive modulation and coding schemes. MIMO is used to increase the overall bitrate through transmission of two (or more) different data streams on two (or more) different antennas using the same resources in both frequency and time, separated only through use of different reference signals to be received by two or more antennas. The enhanced-MIMO techniques take the name of Cooperative Multipoint Transmission (CoMP). It is a strategy to improve users performance is the inter-cell interference reduction. CoMP is based on cooperation between different base stations.

There is a growing interests to interconnect and amalgamate different technologies to support future connectivity and data rates. Make our everyday life more efficient, comfortable and safer is the main objective of the Internet of Things. Services such as smart mobility, smart environment, and e-health will continue to proliferate and become more mobile.

The evolving of cellular wireless networks, fifth generation (5G), are envisioned to overcome the fundamental challenges of existing cellular networks, e.g., higher data rates, user-coverage and crowded areas, energy consumption and cost efficiency. 5G wireless networks are expected to be a mixture of network tiers of different sizes, a multi-tier architecture consisting of macro-cells, different types of licensed small-cells, device-to-device (D2D) networks to serve users with different quality-of-service (QoS) and different radio access technologies (RATs) that are accessed by an unprecedented numbers of smart and heterogeneous wireless devices [7].

The deployment of small-cells bring an improvement in order of network coverage and due to the changes to the functional architecture of the access network allowed data and control signals to tunnel through the Internet, enabling small-cells to be deployed anywhere with Internet connectivity [8]. Small-cells are also a very promising candidate for the backhauling of WSNs (Wireless Sensor Networks), because terminals can use less power in comparison with other wireless systems [9]. Recent developments are moving the WSN communication towards Internet protocol (IP)-based systems, according to the IoT paradigm. 
The vision of the future $5 \mathrm{G}$ seems to be a heterogeneous networks composed of a mixture of different radio access technologies that include WLAN technologies which can offer seamless handovers to and from the cellular infrastructure, and device to device communications.

Another new important aspect where the next generations networks are focusing are the "big data". The future M2M or IoT applications will generate a vast amount of data and obviously will be a technical challenge for RANs. New network architectures may emerge from the necessity of running big data applications, making informed decisions and extracting intelligence from big data. The smart grid can be seen as a huge sensor network, with immense amounts of grid sensor data from various sensors, meters, appliances and electrical vehicles. Data mining and machine learning techniques are essential for efficient and optimized operation of the grid [8].

\begin{tabular}{|c|c|c|c|c|c|c|c|}
\hline Generations & $1 G$ & $2 \mathrm{G}$ & $2.5 \mathrm{G}$ & $3 G$ & $3.5 \mathrm{G}$ & $4 \mathrm{G}$ & $5 G$ \\
\hline $\begin{array}{c}\text { Start/Deployme } \\
\text { nt }\end{array}$ & $\begin{array}{l}1970- \\
1980\end{array}$ & $1990-2004$ & $\begin{array}{l}2001- \\
2004\end{array}$ & $2004-2010$ & $\begin{array}{l}2006- \\
2010\end{array}$ & 2010-Now & Soon \\
\hline Data Bandwidth & $2 \mathrm{Kbps}$ & 9.6- $43.2 \mathrm{Kbps}$ & $\begin{array}{c}144- \\
384 \\
\text { Kbps }\end{array}$ & $2 \mathrm{Mbps}$ & $\begin{array}{l}\text { More } \\
\text { than } \\
2 \mathrm{Mbps}\end{array}$ & $1 \mathrm{Gbps}$ & $\begin{array}{l}\text { Higher } \\
\text { than } \\
\text { 1Gbps }\end{array}$ \\
\hline Technology & $\begin{array}{c}\text { Analog } \\
\text { Cellular } \\
\text { Technolog } \\
\text { y }\end{array}$ & $\begin{array}{c}\text { Digital } \\
\text { CellularTechnolo } \\
\text { gy }\end{array}$ & $\begin{array}{c}\text { GPRS, } \\
\text { EDGE } \\
\text { ' } \\
\text { CDM } \\
\text { A }\end{array}$ & $\begin{array}{c}\text { CDMA } \\
\text { 2000(1xRTT } \\
, \\
\text { EVDO)UMT } \\
\text { S, EDGE }\end{array}$ & $\begin{array}{l}\text { EDGE, } \\
\text { Wi-Fi }\end{array}$ & $\begin{array}{l}\text { Wi-Max, } \\
\text { LTE, LTE-A, } \\
\text { Wi-Fi }\end{array}$ & WWWW \\
\hline Service & $\begin{array}{l}\text { Mobile } \\
\text { Telephon } \\
\text { y (Voice) }\end{array}$ & $\begin{array}{c}\text { Digital voice, } \\
\text { SMS, Higher } \\
\text { capacity } \\
\text { packetized data }\end{array}$ & $\begin{array}{l}\text { SMS, } \\
\text { MMS }\end{array}$ & $\begin{array}{l}\text { Integrated } \\
\text { high quality } \\
\text { audio, } \\
\text { video and } \\
\text { data }\end{array}$ & $\begin{array}{l}\text { Integrate } \\
\text { d high } \\
\text { quality } \\
\text { audio, } \\
\text { video } \\
\text { and data }\end{array}$ & $\begin{array}{c}\text { Dynamic } \\
\text { Informatio } \\
\mathrm{n} \text { access, } \\
\text { Wearable } \\
\text { devices }\end{array}$ & $\begin{array}{c}\text { Dynamic } \\
\text { Informatio } \\
\mathrm{n} \text { access, } \\
\text { Wearable } \\
\text { devices } \\
\text { with Al } \\
\text { Capabilitie } \\
\mathrm{s}\end{array}$ \\
\hline Multiplexing & FDMA & TDMA, CDMA & $\begin{array}{c}\mathrm{CDM} \\
\mathrm{A}\end{array}$ & CDMA & CDMA & $\begin{array}{l}\text { OFDMA, } \\
\text { SCFDMA }\end{array}$ & $\begin{array}{l}\text { OFDMA, } \\
\text { SCFDMA }\end{array}$ \\
\hline Switching & Circuit & Circuit, Packet & $\begin{array}{c}\text { Packe } \\
t\end{array}$ & Packet & $\begin{array}{c}\text { All } \\
\text { Packet }\end{array}$ & All Packet & All Packet \\
\hline Core Network & PSTN & PSTN & PSTN & $\begin{array}{l}\text { Packet } \\
\text { N/W }\end{array}$ & Internet & Internet & Internet \\
\hline
\end{tabular}

Table 2: Generations of mobile technologies [10].

3. Modeling and analysis of interference in the heterogeneous wireless networks 
In parallel with cellular system development, the wireless technologies sector provides different access systems: Wireless Local Area Networks (WLAN) 802.11 IEEE standard (Institute of Electrical \& Electronics Engineers) and Wireless Metropolitan Area Networks (WMAN) 802.16 (commercialized under the name WiMAX from Worldwide Interoperability for Microwave Access). These technologies can be incorporated in the next generation of wireless systems to balance the load of the macro base station and provide a better coverage.In the future wireless networks, small-cells that use these technologies are considered as a promising solution to the increasing number of users of the next generation of wireless networks. Small-cells are equipped with low power and low cost access points which are set up based on the coverage or capacity demand in specific parts of the network and can offload parts of the demand of the macro base station. Coexistence of these technologies in a multi-tier heterogeneous network triggers the need for a more efficient spectrum management schemes to better utilize the spectrum and limit the level of interference from different tiers of the network.

Interference is a major limiting factor in multi-tier heterogeneous networks due to the coexistence of several tiers in the networks. Thus, modeling and analysis of the interference provide insight to the limitations of the network and lead to more practical solutions to respond to the high demand for capacity.Interference in wireless networks is the unwanted signal energy received from peer transmitters on the same network or other nearby networks. The transmitted power $P_{t}$ decays exponentially with respect to the distance $d$ from the transmitter,

$$
P_{r}(d)=K P_{t} h_{r t} d^{-\eta}
$$

where, $P_{r}, K$ and $h_{r t}$ are the received power, path loss constant, and the random channel gain variation between the transmitter and receiver respectively. Signal to noise and interference ratio (SINR) is defined as,

$$
\operatorname{SINR}=\frac{P_{r}(d)}{N+\sum_{i \in \mathbb{I}} P_{r}\left(d_{i}\right)},
$$

where, $N$ is the noise power. $d_{i}$ and $P_{r}\left(d_{i}\right)$ are the distance and received power between the receiver and the $\mathrm{i}^{\text {th }}$ transmitter in the set of nearby transmitters (II) respectively.

Legacy analytical methods for cellular wireless networks assumed hexagonal grids to model the base station (BS) locations and the coverage in the network. However, in practice, placement of the BSs is not regular. This is because the placement is performed based on the demand in specific areas and also the condition of the location and other barriers. In addition, placing small-cells would further randomize the network grid in the next generation wireless networks. Stochastic geometry is a mathematical tool that can provide the expected values of the desired 
parameters in a network of randomly distributed points. Stochastic geometry has been used to model and analyze ad-hoc wireless networks for nearly three decades [11-18]. It has been shown that stochastic geometry can be used for modeling and analysis of the next generation multi-tier wireless networks as well [19-23].

In stochastic geometry models, a network is modeled by a point process that best fits the network characteristics. e.g. large networks in vast areas of coverage and randomly distributed nodes such as cellular networks can be modeled by Poisson point process (PPP). A point process is Poisson point process if and only if the number of points in any compact set defined on the region is a Poisson random variable. Other point processes have also been used to model wireless networks. Binomial point process (BPP) is used to model a randomly distributed wireless sensor network where the number of nodes is known andfinite. Poisson cluster process (PCP) is applied to the network where nodes are clustered around specific point due to some physical constraint or MAC protocol specification such as Wi-Finetworks. Hardcore point process (HCPP) is another point process used in the networks where two nodes cannot coexist within a hardcore vicinity $r_{h}$.

Performance metrics of the network can be obtained using the network model. As an example, by assuming Rayleigh fading for point-to-point channel in the network, the cumulative distribution function (CDF) of the signal to noise and interference ratio (SINR) for the test receiver in the network can be calculated as follows [11],

$$
\begin{aligned}
F_{\text {SINR }}(\theta) & =P\{S I N R \leq \theta\}=P\left\{\frac{K P_{t} h_{r t} d^{-\eta}}{N+I} \leq \theta\right\} \\
& =P\left\{h_{r t} \leq \frac{(N+I) \theta d^{\eta}}{K P_{t}}\right\} \\
& =\int_{u} F_{h_{r t}}\left(\frac{(N+I) \theta d^{\eta}}{K P_{t}}\right) f_{I}(I) d I
\end{aligned}
$$

substituting Rayleigh CDF yields,

$$
F_{S I N R}=1-\left.\exp \left(-\frac{N \mu \theta d^{\eta}}{K P_{t}}\right) \mathcal{L}_{I}(s)\right|_{s=\frac{\mu \theta d \eta}{K P_{t}}}
$$


where,

$$
\operatorname{SINR}=\frac{K P_{t} h_{r t} d^{-\eta}}{N+I}
$$

$F_{h_{r t}}$ and $f_{I}$ are the cumulative distribution function and probability distribution function of the channel and interference respectively. $\mathcal{L}_{I}(s)$ is the Laplace transform of the probability distribution function (PDF) of the interference and is obtained based on the statistics of the point process that fits the network such as Poisson point process [24]. This method yields the CDF of SINR and hence the statistics of the other parameters of the systems such as the outage probability and maximum achievable rate.

In the same way, the average transmission rate in the network could be calculated. In [24], the average transmission rate for the downlink and in Rayleigh fading is calculated. BSs and users are modeled by homogenous PPPs. It is shown that the average transmission rate can be obtained as,

$$
E(\ln (1+S I N R))=\int_{0}^{\infty} e^{-\frac{N \mu d^{\eta}}{K P t}\left(e^{t}-1\right)} \mathcal{L}_{I}\left(\frac{\mu d^{\eta}}{K P_{t}}\left(e^{t}-1\right)\right) d t
$$

However, this method only applies for Rayleigh fading while in general the PDF of SINR may not be obtained in closed form. Another method used in literature is to obtain a lower bound using the major interference contributors. In high path loss environments $(\eta=4)$ the nearest $n$ interferers can be included into the interference calculations where $n$ is the parameter. e.g. to obtain a lower bound for the outage probability, the vulnerability region is defined such that existence of a transmitter within this region would drive the SINR below a desired threshold $\theta$. The probability that at least a transmitter is present in vulnerability region is then calculated [21].

Frequency reuse technique is widely used in cellular networks to mitigate the interference experienced by the users at the expense of lower spatial bandwidth efficiency. In generic frequency reuse scheme, the total available bandwidth is divided into $\Delta$ sub-bands and used by different cells in a way that no two neighboring cells would use the same sub-band.Fractional frequency reuse (FFR) can improve the spatial frequency bandwidth efficiency by reusing a large portion of the available bandwidth for their inner cell users while assigning the rest to the borderline users. This is performed by assigning transmission power $\mathrm{P}_{1}$ to the inner cell frequencies and $P_{2}$ to the boundary frequencies where $P_{2}>P_{1}$. Obviously, the neighboring cells would not use will not use the same boundary frequencies. 
In general, incorporating frequency reuse schemes in stochastic geometry network models is a challenging task, as frequency reuse will violate the fundamental spatial in independency in PPP. This is because, the base stations with the same set of sub-band cannot be neighbors and hence their locations are correlated. A solution is to assume that the BSs randomly choose their sub-bands. Hence, cells using the same sub-band will form a thinned version of the original PPP which is also a PPP with $\frac{\lambda}{\Delta}$ where $\lambda$ is the intensity of the nodes in the original PPP and $\Delta$ is the number of sub-bands. Incorporating FFR into the network model is tricky due to the correlation it introduces to the placement of the BSs using similar boundary frequencies. This has been overcome in [25] by introducing a Threshold $\mathrm{T}_{\text {ffr }}$ where users with SINR lower than threshold are considered boundary users.

Using small-cells in multi-tier next generation cellular networks improves the network coverage and capacity. Spectrum allocation to the small-cells can be universal frequency reuse or by spectrum partitioning. In universal frequency reuse, the whole spectrum is used by macro-cells and small-cells. This improves the spatial spectrum efficiency at the cost of multi-tier interference. i.e. small-cell users will receive interference from macro-cell and vice versa. On the other hand, spectrum partitioning will eliminate the multi-tier interference at the expense of lower spectrum efficiency. This is because, only parts of spectrum is used at every tier of the network. In [26], hexagonal grid model is adopted for macro-cells while small-cells are modeled by PPP and the optimum spectrum partitioning is investigated. The trade-off between spectrum sharing and partitioning is investigated in [19]. All network tiers are modeled by PPP and Rayleigh fading is assumed for channel fading. Results show that universal spectrum sharing is optimal in sparse network deployments while spectrum partitioning is more desired in dense networks in terms of transmission capacity and outage probability.

Using centralized scheduling for spectrum access in future multi-tier wireless network is inefficient in terms of delay and complexity. Hence, cognitive radio and opportunistic medium access are considered viable solutions for this type of networks. Modeling and analyzing interference is crucial in this type of networks as interference is considered a major limiting factor of the efficiency of this type of networks.

Electromagnetic spectrum is a limited natural resource and most of the usable bands are already licensed for specific applications. In addition, a small portion of the spectrum has been released by authorities for industrial, scientific and medical purposes. However, these license free bands are increasingly getting over-populated and hence they have a high level of interference. Nevertheless, study shows that the licensed spectrum is under-utilized by the licensed operators. In other words, there are spatial or temporal white spaces where the spectrum is not used by the primary licensed user. Cognitive radio is a term assigned to the set of schemes where the wireless device can sense and adjust its parameters for a more efficient 
use of the spectrum. In future, cognitive radio devices will be allowed to access the licensed spectrum as a secondary user, conditioned on limited interference to the primary user. However, before these dynamic sharing schemes can be used in practice, their spectrum efficiency and impact on the primary users must be carefully analyzed as discussed in Chapter XX. [Reference to the chapter "Wireless Cognitive Networks Technologies and Protocols"]

Stochastic geometry models can be used to evaluate the performance of cognitive radio networks. In [27], the aggregate interference by secondary user received at the primary user is obtained. The primary user network consists of one transmitter and one receiver while secondary user network is modeled by a PPP. Candidate distributions are tested against the interference model and shifted log-normal distribution is suggested as the best distribution describing the statistics of the interference. Similarly, in [28], the interference from a secondary user on a one link primary user is investigated. The probability distribution function (pdf) of interference is obtained through its characteristic function. Results show that a truncated alpha-stable function best represents the pdf of aggregate interference at the primary network receiver. The outage probability of the primary user is obtained by assuming Rayleigh fading for the links and PPP for the distribution of the node in secondary user network. In addition, lower bounds on the interference temperature are obtained using the strongest interferers. The abovementioned works assume a simple one-link network for the primary user. A more complex network model for the primary user network is investigated in [29]. Two independent PPPs are considered for primary and secondary users. Two approaches are considered to obtain the statistics of the outage and interference. In the first approach, bounds for theinterference and outage are obtained by assuming a bipolar Poissoncognitive network model. Whereas, in the second approach the secondary user is assumed to transmit only outside the exclusion region where the received power from the primary transmitters is below a certain threshold. It is shown that the network of the secondary user outside the exclusion region can be accurately modeled by a Poisson hole process[29]. However, due to the complexity of the Poisson hole process, the secondary user network model is approximated and analyzed by Poisson cluster process.

Multi-tier structure of the next generation wireless networks leads to a better coverage and more spectral efficiency. However, a centralized coordination of spectrum access for all tiers of the network is not an efficient solution for spectrum access management of the total network. Femto-cells are envisioned to be installed by the user or operators to respond to the demand for coverage in specific areas and hence their distribution tends to be random. Cognitive radio techniques have been investigated as a promising solution for a de-centralized spectrum access management in such multi-tier cellular networks. In [20], the aggregate interference for a twotier cellular network consists of a single MBS and a network of femto-cells is obtained through its characteristic function. In their proposed scheme, MBS will transmit a busy tone to reserve 
the channel and hence femto-cells will defer their transmission if the received power from MBS is above a certain threshold. The outage probability and the average transmission rate areobtained through the aggregate interference statistics and the model is validated through simulations.

In [21], a more complex network model is investigated. The network is composed of two tiers where users are being served by multiple MBSs and femto-cells. Base stations, access points and users are modeled by independent PPPs. It is shown that cognitive techniques can decrease the probability of outage for femto-cells users by 60 percent. In [22], fully cognitive and semi cognitive schemes are investigate for femto-cells in a cellular network. In semi cognitive technique, the femto-cell access point avoid using the channel occupied by MBSs and aggressively use the second-tier frequencies while in fully cognitive scheme the access points sense and avoid using the frequencies used by other femto cells as well. It is shown that semi cognitive scheme outperforms the fully cognitive scheme in terms of outage probability. This is because although cognition with respect to all network tiers reduces the interference level, it limits the transmission opportunity severely in dense networks.

\section{Simulation techniques for the next generation wireless heterogeneous networks}

Due to the data traffic demand in cellular networks, improvements in system spectral efficiency are necessary. One possible solution is increasing the base station deployment density. In a relatively sparse deployment of macro base stations, adding another base station does not severely affect inter-cell interference, and solid cell splitting gains are easy to achieve.However, site acquisition in a capacity limited dense urban area can get prohibitively expensive [30].

Challenges associated with the deployment of traditional macro base stations can be overcome by the utilization of base stations with lower transmit power which are classified as pico-cells, femto-cells and relay nodes. The transmit power of the low power access points intended for outdoor deployments ranges from $250 \mathrm{~mW}$ to approximately $2 \mathrm{~W}$. They do not require an air conditioning unit for the power amplifier and are much lower in cost than traditional macro base stations, which their transmit power typically varies between 5 and $40 \mathrm{~W}$. Pico-cells are regular eNBs with the only difference of having lower transmit power than traditional macrocells. They are, typically, equipped with Omni-directional antennas and are deployed indoors or outdoors often in a planned (hot-spot) manner. Femto base stations are meant for indoor use, and their transmit power is typically $100 \mathrm{~mW}$ or less. Unlike pico, femto base stations may be configured with a restricted association, allowing access only to its closed subscriber group (CSG) members. Such femto base stations are commonly referred to as closed femtos. Relay nodes are used to extend the macro-cell coverage or fill a coverage hole. A network that consists of a mix of macro-cells and low-power nodes, where some may be configured with 
restricted access and some may even lack wired backhaul, is referred to as a heterogeneous network. An illustration of such networks is shown in Figure 1,

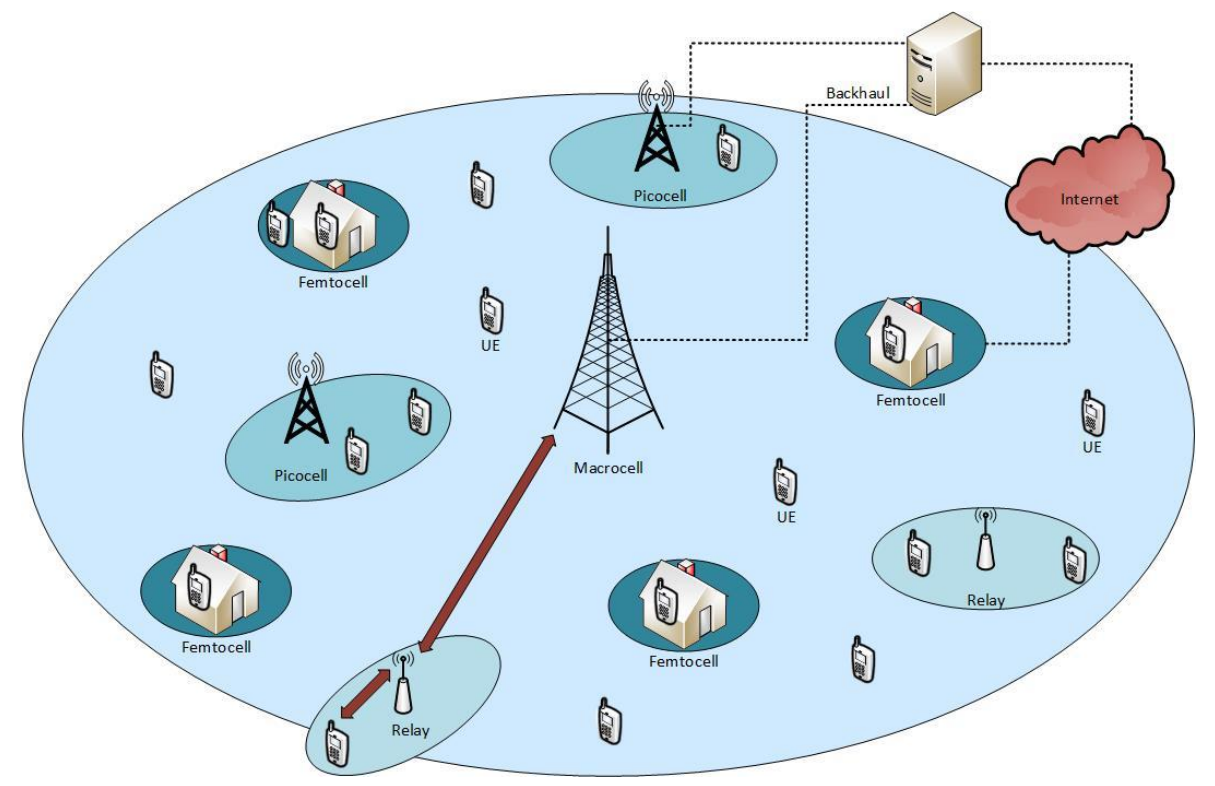

Figure 1: An illustration of a heterogeneous network

Femto-cells have recently attracted significant attention. The use of femto-cells will benefit both users and operators. Users will enjoy better signal quality due to the proximity between transmitter and receiver and hence communicate with larger reliabilities and throughputs. Furthermore this will also provide power savings, reduce electromagnetic interference and energy consumption. This way, more users will access to the same pool of radio resources or use larger modulation and coding schemes, while operators will benefit from greater network capacity and spectral efficiency. There are a number of technical studies associated with various aspects of femto-cells deployments based on cellular technology. These studies consider operations, administration, and management (OAM) and self-organizing network (SON) protocols, network architecture, local IP access (LIPA), access (open, closed, and hybrid), and interference management [31-33].

Heterogeneous networks are based on different wireless technologies, where macro networks are based on a cellular technology while low power access points are based on WLAN [34-35]. Reduced cost is one of the main motives for the adoption of femto-cells. It is shown in [36] that in urban areas a combination of publicly accessible home base stations or femto-cells (randomly deployed by the end user), and macro-cells deployed by an operator for area coverage in a planned manner, can result in significant reductions (up to 70 percent in the investigated scenario) of the total annual network costs compared to a pure macro-cellular network deployment. 
The introduction of low-power nodes in a macro network creates imbalance between uplink and downlink coverage. Due to larger transmit power of the macro base station, the handover boundary is shifted closer to the low-power node, which can lead to severe uplink interference problems as UE units served by macro base stations create strong interference to the lowpower nodes.

The performance of a mixed deployment of macro-, pico-, and open femto-cells was evaluated in [37-38], which showed that the limited coverage of low-power nodes is the main reason for limited performance gain in heterogeneous networks.

In 4G networks, new physical layer design allows for flexible time and frequency resource partitioning. This added flexibility enables macro-, femto- and pico-cells to assign different time-frequency resource blocks within a carrier or different carriers (if available) to their respective UE. This is one of the inter-cell interference coordination (ICIC) techniques that can be used on the downlink to mitigate data interference [39-40]. With additional complexity, joint processing of serving and interfering base station signals could further improve the performance of heterogeneous networks [41-42], but these techniques require further study for the scenarios commonly seen in practice.

\subsection{NS-3 Simulator}

ns-3 (network simulator 3 ) is a discrete time event based network simulator. It can implement a large number of different protocols and it is completely free software, licensed under GNU GPLV2 license. ns-3 is a standalone new simulator and it is not an extension of ns-2. The two simulators are both written in $\mathrm{C}++$, but ns-3 does not support the ns-2 APIs. Some models from ns-2 have already been adapted for use in ns-3. Both ns-2 and ns-3 are discrete event network simulators. This means that the simulation consists of a series of independent events that change their state. Events are actions such as a sending a packet, a new node being added to the network, or a timer expiring.

Each scheduled event runs until completion without advancing the simulation time, and then the simulation time is increased to the start time for the next scheduled event.

ns-3 is developed mostly by the same group of people that work on or have worked on ns- 2 [43]. The ns-3 project started because of some problems associated with the ns-2. In ns-2, C++ and $\mathrm{tcl}$ are used to build simulations and the combination of both languages is difficult to debug and it can be considered as a barrier for new developers. Different tests show that ns- 2 does not scale well to large simulations, making it unsuitable for some research scenarios. Many models in ns-2 are not validated against the real world, which makes users to doubt whether simulation results are the same as the results that would be measured in real-world implementations. 
The nodes in the simulation with ns-3 are more realistic than ns-2. Every node is constructed out of devices and there is an Internet protocol stack that closely resembles the stack on real systems.

Network traffic generated by ns-3 can be traced and written to a file in the pcap (packet capture) format, which makes it possible to analyze it with tools like "Wireshark".

Due to its ability to simulate realistic scenarios, ns-3 can be used as a virtual system or be used in testbeds. Real applications can run on top of a protocol stack implemented by ns-3 and it is also possible to run applications on real networking stacks or to run an instance of ns-3 in a network, where it interacts with 'real' systems.

The ns-3 architecture consists of a core simulator part and a number of layers that add the networking-specific elements. It provides an Internet stack with implementations of protocols like TCP and UDP, as well as lower-level protocols such as various versions of 802.11. Different components and applications can be added to the nodes, after which nodes can be connected to each other. To help with building up nodes and creating a network topology, helper scripts are provided. Compared to ns-2, there are other differences as well, such as the build system.

The simulation core is implemented in src/core. Packets are fundamental objects in a network simulator and are implemented in src/network. These two simulation modules by themselves are intended to comprise a generic simulation core that can be used by different kinds of networks, not just Internet-based networks.

The first open source product-oriented LTE network simulator is developed by Ubiquisys, the developer of intelligent cells, and the Centre Tecnologic de Telecomunicacions de Catalunya (CTTC) [44]. The development of the LTE module for the ns-3 was carried out during the Google Summer of Code 2010. This module provides a basic implementation of the LTE device, including propagation models, PHY and MAC layers. The simulator will provide a common platform for LTE femto- and macro-cells. In WCDMA networks, femto-cells and macro-cells work independently, but in LTE all cells work together as a single self-organizing network. This means that the adaptive behavior of femto-cells and macro-cells is interdependent. Simulations are important because they can evaluate product performance in densely deployed and heavily used networks while real deployments are still in their infancy. The development of the LTE simulator is open to the community in order to foster early adoption and contributions by industrial and academic partners. The most important features provided by this module are: a basic implementation of both the User Equipment (UE) and enhanced NodeB (eNB) devices, 
Radio Resource Control (RRC) entities for both the UE and the eNB, Adapting Modulation and Coding (AMC) scheme for the downlink, the management of the data radio bearers, Channel Quality Indicator (CQI) management, etc.

In LTE network, a module consists of two main components:

- The LTE Model. This model includes the LTE Radio Protocol stack (RRC, PDCP, RLC, MAC, PHY). These entities reside entirely within the UE and the eNB nodes. This has been designed to support the evaluation of the Radio Resource Management, QoS-aware Packet Scheduling, Inter-cell Interference Coordination, and Dynamic Spectrum Access;

- The EPC (Evolved Packet Core) Model. This model includes core network interfaces, protocols and entities. These entities and protocols reside within the SGW, PGW and MME nodes, and partially within the eNB nodes. The EPC model provides means for the simulation of end-to-end IP connectivity over the LTE model. In particular, it supports for the interconnection of multiple UEs to the Internet, via a radio access network of multiple eNBs connected to a single SGW/PGW node.

\subsection{Simulations}

In this section some example scenarios are simulated using ns-3 network simulator and the results will be presented and discussed. In the following simulations, the scenarios are implemented in ns-3 as shown in Figure 2.

The scenario includes a single macro-cell, a number of femto-cells and users of both macro-cell and femto-cell. Femto-cell coverage is CSG (Closed Subscriber Group) where only the authorized users can access the base station. Figure 2 shows a HetNet composed of a macrocell (eNB), different femto-cells (HeNB) and users where MUE (macro-user equipment) and HUE (home-user equipment) indicate macro- and femto-cell users respectively. Also the core network or EPC has been implemented, following procedures explained in [31]. Moreover, different applications are considered to generate user traffic in downlink and uplink. LTE provides users traffic with different QoS (Quality of Serivce). Each information flow is associated with a specific QoS class which constitutes a bearer. "Guaranteed Bit Rate Conversational Voice" bearer is used in the following simulations. The built in radio channel model "Multi Model Spectrum Channel" is used for the links, while the Path Loss and Fading are implemented according to the class "ns-3: Hybrid Buildings Propagation Loss Model". Finally, the UE mobility are incorporated using the "Mobility Model" class. 


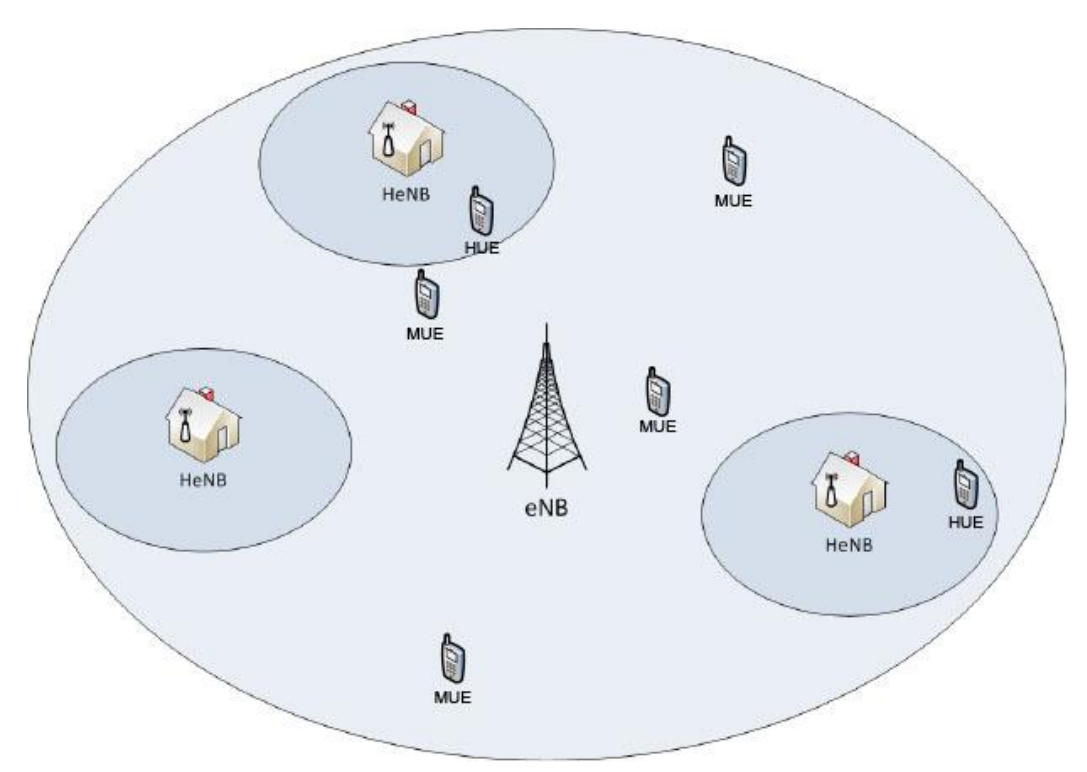

Figure 2: Simulation scenario

The EPC network has the following parameters:

- Data Rate: $100 \mathrm{~Gb} / \mathrm{s}$;

- Delay: $10 \mathrm{~ms}$;

- Maximum Transmission Unit (MTU): 1500 byte.

The LTE network is formed by eNB, HeNB and their respective UE that has these parameters:

- Bandwidth in downlink, measured in number of Resource Blocks (RB): 25;

- E-UTRAN Absolute Radio Frequency Channel Number (EARFCN) in downlink: 100;

- Transmission Power: $10 \mathrm{dBm}$ in the $\mathrm{EU}, 46 \mathrm{dBm}$ in eNB and $20 \mathrm{~dB}$ in HeNB.

The simulation time was set at 15 seconds and the packet size was 1000 bytes (1030 with the PDCP header).

The Radio Environment Map (REM) as shown in Figure 3 is a uniform 2D grid of values that represent the Signal-to-Noise ratio in the downlink with respect to the eNB that has the strongest signal at each point. Through the use of this map, we can better examine the tested scenarios, with respect to the possible interference between devices. 


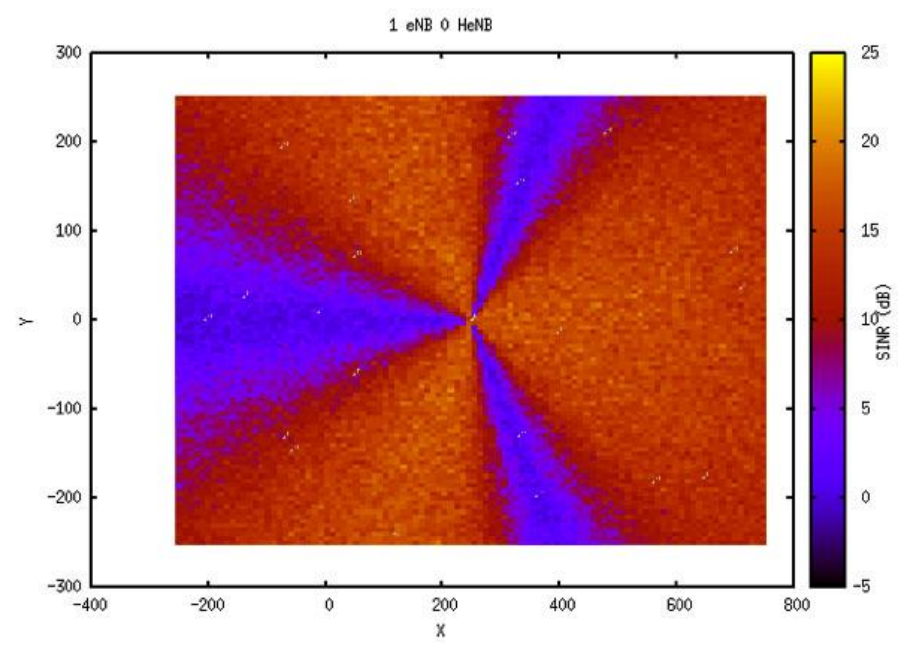

Figure 3: REM map with only one eNB without HeNB

Simulation results presented below are referred to the metrics identified at the PDCP layer. For this set of simulations a throughput metric is taken into account, defined as the number of successful received packets during a specific time. Furthermore, two different kind of throughput are defined: the total throughput and the average throughput.

The total throughput represents the amount of total received information per unit of time and is calculated as,

$$
\text { total throughput }=\frac{R_{x}}{T_{\text {sim }}}
$$

where $R_{x}$ represents the total received number of bits and $T_{\text {sim }}$ is the time of transmission of the bits.

The average throughput is instead expressed by the following equation (1.2):

$$
\text { average throughput }=\frac{R_{x}}{T_{\text {sim }} \cdot N_{\text {Users }}},
$$

which represents the amount of information received by the users per unit of time. $N_{U s e r s}$ is the total number of users. 


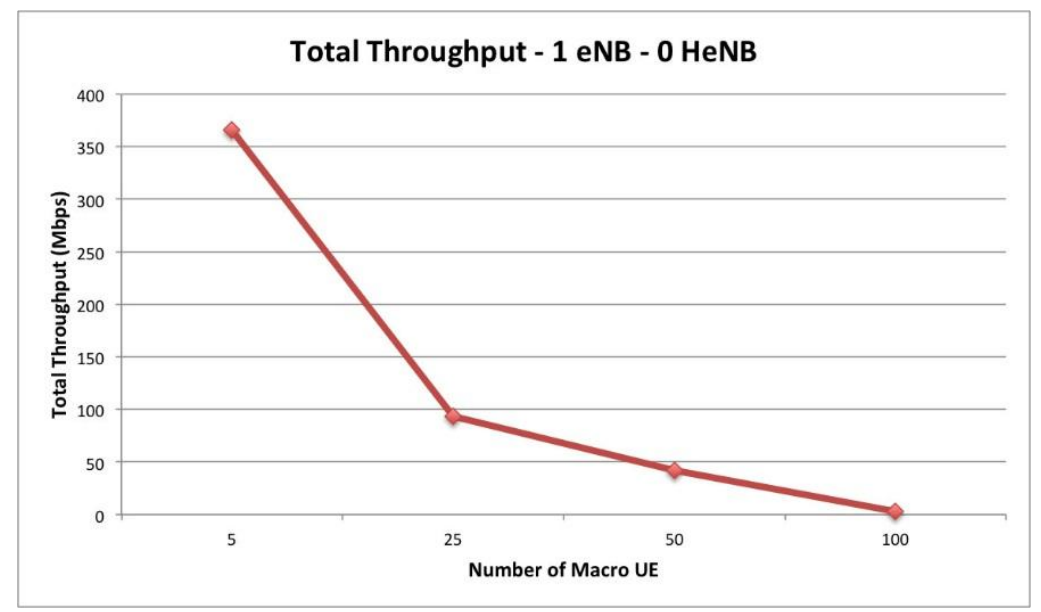

Figure 4: The total throughput for the same scenario

Figure 4 shows the total throughput vs. the number of Macro-cell users when there is no femto-cell. This configuration is chosen to evaluate the throughput vs. the number of UEs. As shown in the figure, increasing the number of users decreases the throughput. This is due tothe increase in the interference received from other users. Further simulations are conducted considering a single femto-cell, HeNB as shown in Figure 4.

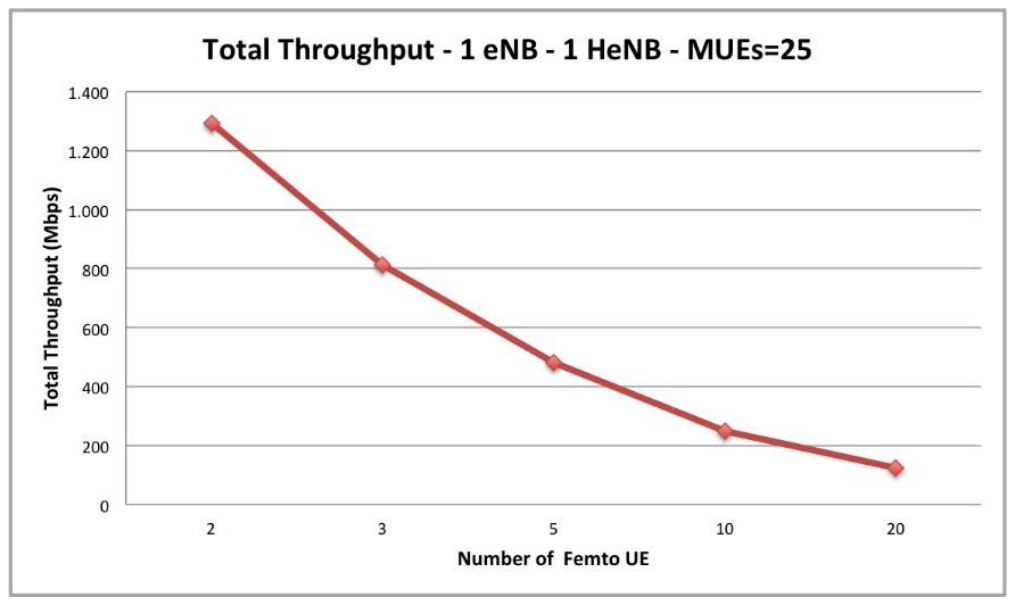

Figure 5: Total throughput in a network with an eNB, HeNB and the number of macro users set to 25 (The number of users of the femto-cell has been varied).

As shown in the Figure 5 the total throughput when 25 UEs are allocated in the macro-cell and the remaining are assigned to one femto-cell, has similar trend as observed in the previous simulations. 
On the other hand, when the users decide on connecting the femto-cell and macro-cell base stations based on the power reception (load balancing) the network throughput is increased as shown in Figure 5.

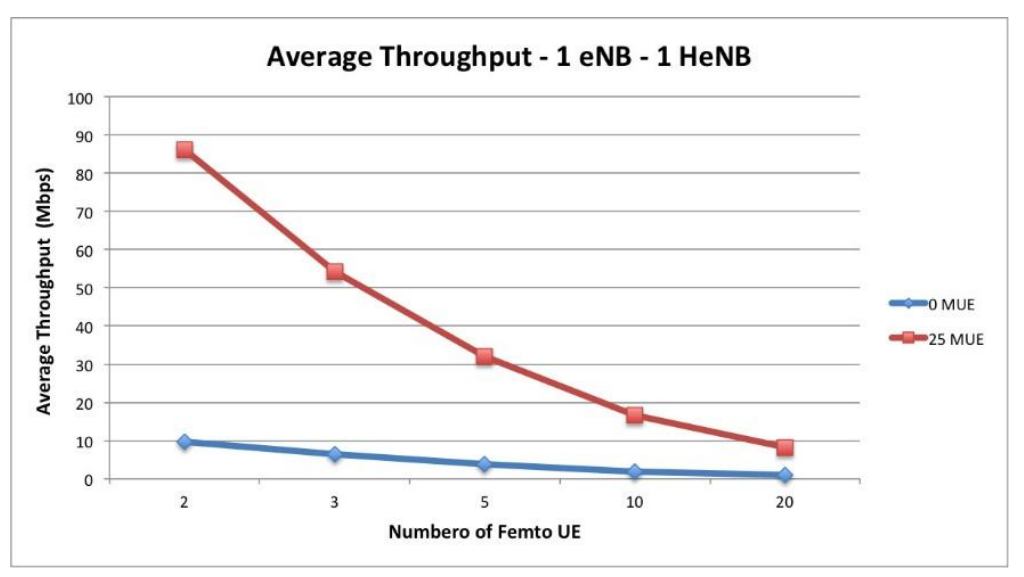

Figure 6: Comparison between the two scenarios with macro UE equal to 0 and 25 respectively.

The final scenario includes a HetNet with the presence of multiple femto-cells. The number of macro-cell users was fixed at 20. Figure 7 highlights how the throughput improves by increasing the number of femto-cells inside the same macro-cell. As figure shows, with 2 femto-cells, increasing the number of femto UE (greater than 10 HUE) causes a drastic decrease in the throughput while increasing the number of femto-cells to 5 improves the network throughput as more users can be covered. However, the presence of more than 5 femto-cells causes interference in the HetNet system and hence degrades the SINR value and consequently the throughput.

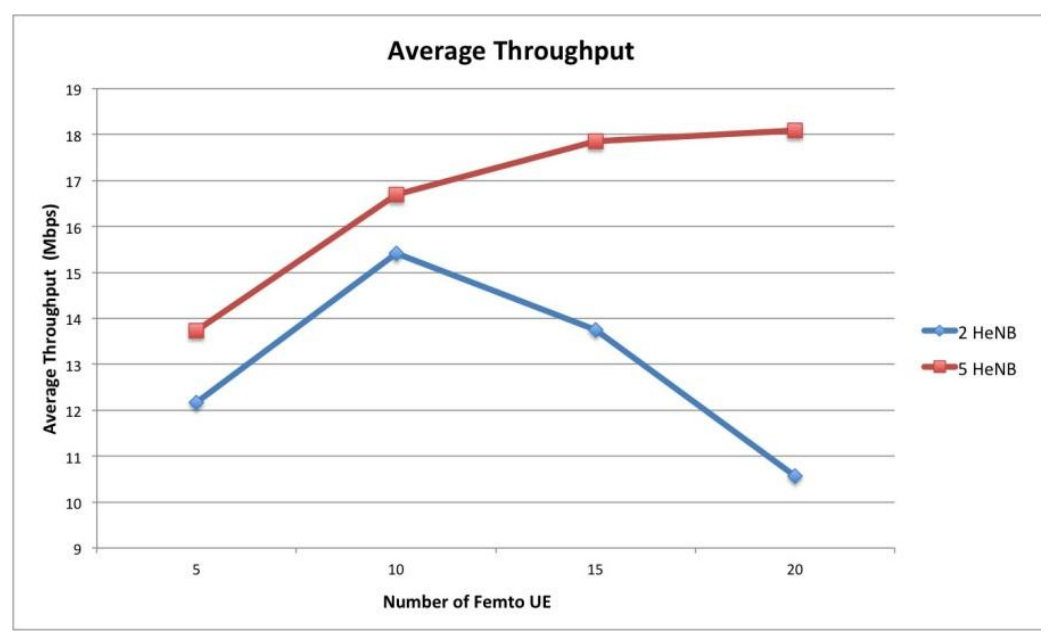

Figure 7: Comparison of average throughput in a scenario with 2 and 5 HeNB by varying the number of HUE 
This is shown in Figure 8. As shown in the figure, increasing the number of femto-cells (more than 5) does not introduce further improvement in terms of throughput due to the increased level of intra-tier interference.

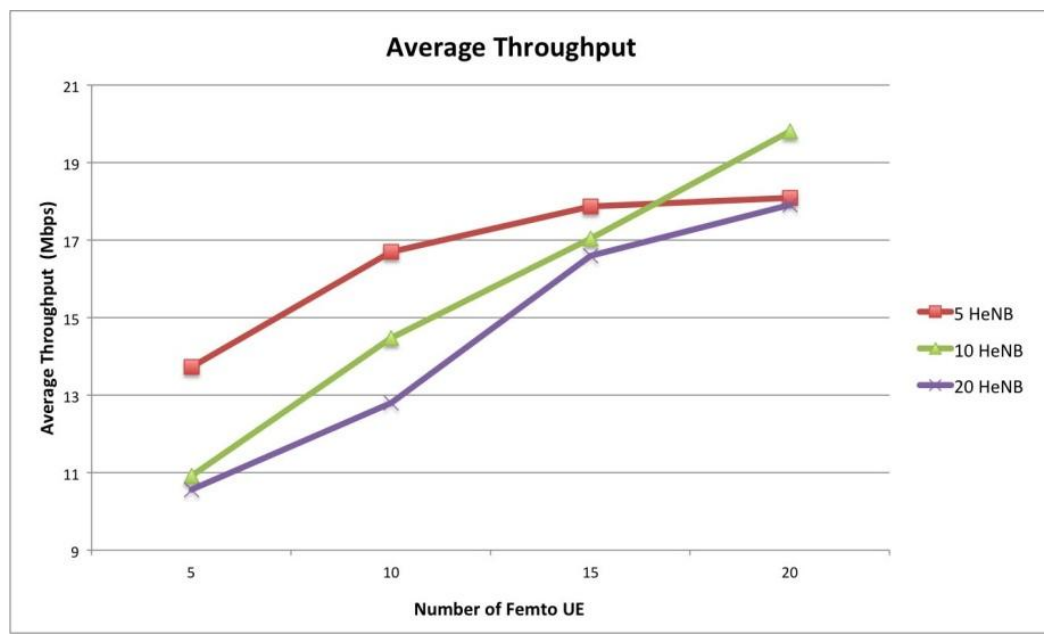

Figure 8: Comparison of the average throughput in a scenario with 5, 10 and $20 \mathrm{HeNB}$ by varying the number of HUE

\section{Conclusion}

Spectrum is a limited natural resource and hence has to be utilized efficiently to support the increasing spectrum demand. Studies show that the spectrum is underutilized by the licensed users. This triggered tremendous interest in using white spaces where the primary user is not accessing the spectrum in spectrum in spatial, temporal or frequency domains. Heterogeneous networks (HetNet) are promising solution to the challenge of providing coverage for a large number of users within the limited available spectrum. Such networks consist of multiple tiers where each tier can use different communication technology, transmission powerand coverage range.Coexistence of these tiers arise some challenges including the interference level which is a major limiting factor in the performance of the next generation multi-tier networks. In this chapter, mathematical models to analyze the interference in such networks and subsequently the throughput are presented. Following the mathematical models, simulation techniques for these networks are discussed. HetNet performance is investigated to testify the improvements introduced by Long Term Evolution-Advanced (LTE-A) definition. The simulation campaign has been conducted with the aim to demonstrate the pros and cons introduced by the use of the femto-cells in a HetNet scenario. Preliminary results have been obtained exploiting the ns-3, building a scenario in which macro-cells and femto-cells coexist has been implemented, in order to evaluate the performance in terms of throughput. Obtained results show that, in general, by increasing the number of femto-cells the system throughput. 


\section{References}

[1] T. Nakamura, S. Nagata, A. Benjebbour, Y. Kishiyama, H. Tang, X. Shen, N. Yang, and N. Li, "Trends in Small Cell Enhancements in LTE Advanced," IEEE Communications Magazine, vol. 51, no. 2, pp. 98-105, Feb 2013.

[2] E. Dahlman, S. Parkvall, and J. Sköld, "4G LTE/LTE-Advanced for Mobile Broadband." Academic Press, Elsevier, 2011

[3] A. Osseiran, J. Monserrat and W. Mohr, "Mobile and Wireless Communications for IMTAdvanced and Beyond," Wiley \& Sons, 2011

[4] N. Boudriga, O. Hassairi, M.S. Obaidat, Intelligent services integration in mobile ATM networks, in: ACM Symposium on Applied Computing (SAC), ACM, SanAntonio, TX, 1998, pp. 91-97.

[5] 3GPP2 X.S0004-700-E, Version 1.0.0, Wireless Intelligent Networks, March 2004.

[6] 3GPP. "Overview of 3GPP release 8 v.0.1.1". Technical report.

[7] E. Hossain, M. Rasti, H. Tabassum, A. Abdelnasser. "Evolution Towards 5G Multi-tier Cellular Wireless Networks: An Interference Management Perspective". IEEE Wireless Communications, to appear.

[8] Chin, Woon Hau, Zhong Fan, and Russell J. Haines. "Emerging Technologies and Research Challenges for $5 G$ Wireless Networks." IEEE Wireless Communications April 2014.

[9] Cimmino, A., Pecorella, T., Fantacci, R., Granelli, F., Rahman, T. F., Sacchi, C., Carlini, C., Harsh, P. The role of small cell technology in future Smart City applications. Transactions on Emerging Telecommunications Technologies, 25(1), 11-20, 2014.

[10] P. Sharma, Evolution of Mobile Wireless CommunicationNetworks-1G to $5 \mathrm{G}$ as well as Future Prospectiveof Next Generation Communication Network, IJCSMC, Vol. 2, Issue. 8, pg.47 - 53, August 2013

[11] H. ElSawy, E. Hossain, and M. Haenggi, "Stochastic geometry for modeling, analysis, and design of multi-tier and cognitive cellular wireless networks: A survey," IEEE Communications Surveys and Tutorials, vol. 15, July 2013.

[12] M. Haenggi, J. G. Andrews, F. Baccelli, O. Dousse, and M. Franceschetti, "Stochastic Geometry and Random Graphs for the Analysis and Design of Wireless Networks," IEEE Journal on Selected Areas in Communications, vol. 27, pp. 1029-1046, Sept. 2009. 
[13] Cardieri, P., "Modeling Interference in Wireless Ad Hoc Networks," IEEE Communications Surveys \& Tutorials, vol.12, no.4, pp.551,572, 2010.

[14] M. Haenggi and R. Ganti, Interference in Large Wireless Networks, in Foundations and Trends in Networking, NOW Publishers, 2008, vol. 3, no. 2, pp. 127-248.

[15] S. Weber and J. G. Andrews, Transmission Capacity of Wireless Networks in Foundations and Trends in Networking, NOW Publishers, February 2012.

[16] F. Baccelli and B. Blaszczyszyn, Stochastic Geometry and Wireless Networks in Foundations and Trends in Networking, Volume 1, NOW Publishers, 2009.

[17] F. Baccelli and B. Blaszczyszyn, Stochastic Geometry and Wireless Networks in Foundations and Trends in Networking, Volume 2, NOW Publishers, 2009.

[18] L. Kleinrock and J. A. Silvester, "Optimum Transmission Radii for Packet Radio Networks or Why Six is a Magic Number," in Conference Record: National Telecommunication Conference, December 1978, pp. 4.3.1-4.3.5.

[19] W. Cheung, T. Quek, and M. Kountouris, "Throughput Optimization, Spectrum Allocation, and Access Control in Two-tier Femtocell Networks," IEEE J. Sel. Areas Communications, vol. 30, no. 3, pp. 561-574, April 2012.

[20] C. Lima, M. Bennis, and M. Latva-aho, "Coordination Mechanisms for Self-Organizing Femtocells in Two-Tier Coexistence Scenarios," IEEE Trans. Wireless Communications, vol. 11, no. 6, pp. 2212-2223, June 2012.

[21] H. ElSawy and E. Hossain, "Two-Tier HetNets with Cognitive Femtocells: Downlink Performance Modeling and Analysis in a Multi-Channel Environment," IEEE Trans. Mobile Computing, accepted.

[22] H. ElSawy and E. Hossain, "On Cognitive Small Cells in Two-tier Heterogeneous Networks," inProc. $9^{\text {th }}$ Workshop on SpatialStochastic Models for Wireless Networks (SpaSWiN 2013),TsukubaScience City, Japan, May 13-17, 2013.

[23] H. ElSawy, E. Hossain, and D. I. Kim, "HetNets with CognitiveSmall Cells: User Offloading and Distributed Channel AllocationTechniques,"IEEE Commun. Mag., Special Issue on“Heterogeneousand Small Cell Networks (HetSNets), May 2013.

[24] J. Andrews, F. Baccelli, and R. Ganti, "A Tractable Approach to Coverage and Rate in Cellular Networks," IEEE Trans. Communication, vol. 59, no. 11, pp. 3122-3134 November 2011. 
[25] H. Dhillon, R. Ganti, F. Baccelli, and J. Andrews, "Modeling and Analysis of K-Tier Downlink Heterogeneous Cellular Networks," IEEE J. Sel. Areas Communication, vol. 30, no. 3, pp. 550560, April 2012.

[26] V. Chandrasekhar and J. Andrews, "Spectrum Allocation in Tiered Cellular Networks," IEEE Trans. Communications, vol. 57, no. 10, pp. 3059-3068, October 2009.

[27] A. Ghasemi and E. Sousa, "Interference Aggregation in Spectrum Sensing Cognitive Wireless Networks," IEEE J. Sel. Topics Signal Process. , vol. 2, no. 1, pp. 41-56, February 2008.

[28] A. Rabbachin, T. Q. S. Quek, H. Shin, and M. Z. Win, “Cognitive Network Interference," IEEE J. Sel. Areas Communications, vol.29,no.2, pp. 480-493, February 2011.

[29] C.-H. Lee and M. Haenggi, "Interference and Outage in Poisson Cognitive Networks," IEEE Trans. Wireless Communications, vol. 11, pp. 1392-1401, April 2012.

[30] A.Damnjanovic, J.Montojo, Y.Wei, T. Ji, T. Luo, M.Vajapeyam, T.Yoo, O.Song, D.Malladi, A Survey on 3GPP Heterogeneous Networks, IEEE Wireless Communications, June 2011.

[31] M. Yavuz et al., Interference Management and Performance Analysis of UMTS/HSPA+ Femto-cells, IEEE Communications, May 2009.

[32] V. Chandrasekhar, J. G. Andrews, A. Gatherer, Femto-cell Networks: A Survey, IEEE Communications, May 2008

[33] C. Patel, M. Yavuz, and S. Nanda, Femto-cells [Industry Perspectives], IEEE Wireless Commun. May,Oct. 2010.

[34] M. Coupechoux, J-M. Kelif, and P. Godlewski, Network Controlled Joint Radio Resource Management for Heterogeneous Networks, IEEE VTC Spring 2008.

[35] W. Song, H. Jiang, and W. Zhuang, Performance Analysis of the WLAN-First Scheme in Cellular/WLAN Interworking,, IEEE Trans. Wireless Commun., May 2007.

[36] H. Claussen, L.T.W. Ho, and L. G. Samuel, Financial Analysis of a Pico-Cellular Home Network Deployment, IEEE ICC 2007.

[37] T. Nihtila and V. Haikola, HSDPA Performance with Dual Stream MIMO in a Combined Macro-Femto Cell Network, IEEE VTC 2010.

[38] H. R. Karimi et al., Evolution Towards Dynamic Spectrum Sharing in Mobile Communications, IEEE PIMRC 2006. 
[39] G. Boudreau et al., Interference Coordination and Cancellation for 4G Networks, IEEE Commun. Mag., 2009.

[40] A. Khandekar et al., LTE-Advanced: Heterogeneous Networks, European Wireless Conf. 2010.

[41] O.Simeone, E.Erkip, S.Shamai, Robust Transmission and Interference Management For Femto-cells with Unreliable Network Access, IEEE JSAC, Dec. 2010.

[42] S. Annapureddy et al., http://www.ieee-ctw.org/2010/mon/Gorokhov.pdf, 2010 IEEE Commun.Theory Wksp., Cancun, Mexico, 2010.

[43]“ns-3 project description". http://www.nsnam.org/docs/proposal/project.pdf.

[44] “Manual LENA release M8”. http://lena.cttc.es/manual. 